\title{
Towards a System Dynamics MOdeling ME- THOD BASED ON DEMATEL
}

\author{
Fadwa Chaker, Abdellah El Manouar, Mohammed Abdou Janati Idrissi \\ ENSIAS Engineering School, Mohammed V University, Rabat, Morocco
}

\begin{abstract}
If System Dynamics (SD) models are constructed based solely on decision makers' mental models and understanding of the context subject to study, then the resulting systems must necessarily bear some degree of deficiency due to the subjective, limited, and internally inconsistent mental models which led to the conception of these systems. As such, a systematic method for constructing SD models could be essentially helpful in overcoming the biases dictated by the human mind's limited understanding and conceptualization of complex systems. This paper proposes a novel combined method to support SD model construction. The classical Decision Making Trial and Evaluation Laboratory (DEMATEL) technique is used to define causal relationships among variables of a system, and to construct the corresponding Impact Relation Maps (IRMs). The novelty of this paper stems from the use of the resulting total influence matrix to derive the system dynamic's Causal Loop Diagram (CLD) and then define variable weights in the stock-flow chart equations. This new method allows to overcome the subjectivity bias of SD modeling while projecting DEMATEL in a more dynamic simulation environment, which could significantly improve the strategic choices made by analysts and policy makers.
\end{abstract}

\section{KEYWORDS}

System Dynamics modeling, DEMATEL, decision making methods, decision support systems.

\section{INTRODUCTION}

In a world where consumers and businesses are faced with prolific options to satisfy their growing needs, decision making approaches and techniques are getting under increased scrutiny. Management researchers, social psychologists, and decision scientists continue to explore pathways combining as varied disciplines as mathematics, computer programming, social sciences and engineering to guide the Decision Making process. As a result, today's findings highlight some important models used by business analysts, policy makers, and researchers in the decision sciences arena. The existing literature [1-4] highlights a number of DM approaches ranging from mathematical models to fuzzy logic based methods. Several research works combined two or more of the techniques to tackle complex questions. In solving for supply chain management problems, for example, [5] combined two Multi-Criteria Decision Making (MCDM) techniques with a Mathematical Programming (MP) method for a large-scale high-tech firm in Taiwan, [6] used Data Envelopment Analysis (DEA) combined with decision trees and the Neural Networks (NN) Artificial Intelligence (AI) method in a supplier evaluation model, and [7] used fuzzy Analytic Hierarchy Process (AHP) and fuzzy Multi-Objective linear Programming (MOP) in developing a low carbon supply chain.

An equally important input to DM is provided by the System Dynamics (SD) approach. Initially introduced by Jay W. Forrester in the mid-1950s, SD is a methodology for conceptualizing the complex interrelations between elements of a system and assessing how these elements progress within and impact upon the entire system. SD is based on simulation methods and has been used 
in as varied fields as, but not limited to, industrial processes [8], forestry projects management [9], supply chain management [10], and sustainable development [11, 12].

While both DM methods and system dynamics were used to address various DM questions, it was found a lack of information on the combination of both approaches to help in the decision process. Considering this important research gap, this paper presents a hybrid method combining Decision Making Trial and Evaluation Laboratory (DEMATEL) [13], a MCDM technique, and the System Dynamics approach as an extended decision making framework. The method suggests constructing a decision support model which explores the causal relationships between a set of indicators using DEMATEL, and which integrates the DEMATEL outcomes as weights in the SD model relations.

The novelty of this research derives from the proposed approach which has not been ex-plored in the literature and which overcomes the main limitations of SD and DEMATEL while taking advantage of their respective strengths. This novel hybrid model not only provides a systematic method for constructing SD models which usually present the shortcoming of subjectivity, but it also allows decision makers to project DEMATEL's fixed results into a time-related environment where it is possible to run simulations and assess the impact of today's strategic choices on tomorrow's overall organizational performance.

This paper is organized as follows: In Section 2 we present the major paradigms in simulation modeling, then we introduce in Section 3 the system dynamics approach and a brief note on the potential limitations of creating SD models based solely on human mental models. Section 4 presents our proposed hybrid SD modeling method. Sub-section 4.1 presents DEMATEL and its revised version [14], while Sub-section 4.2 builds upon DEMATEL to explain our proposed method. An illustration and discussion of the proposed method are presented in Sub-section 4.3. In Section 5, we conclude and close with some research perspectives.

\section{Major Paradigms in Simulation Modeling}

In solving for complex systems, the first step consists of understanding and describing the system. Then, this understanding is conceptualized into a model involving rules, equations, and logical algorithms which serve as the simulation's main inputs. Simulation results are then analyzed and recommendations on alternative solutions are made (eg. Figure 1).

\begin{tabular}{|c|c|c|c|c|}
\hline I. Understand & II. Conceptualize & III. Simulate & IV. Analyze & V. Advise \\
\hline $\begin{array}{l}\text { Describe the } \\
\text { system }\end{array}$ & $\begin{array}{l}\text { Define algorithms, } \\
\text { equations and } \\
\text { logical formulas }\end{array}$ & $\begin{array}{l}\text { Check the } \\
\text { model, run } \\
\text { under various } \\
\text { scenarios }\end{array}$ & $\begin{array}{l}\text { Analyze, } \\
\text { discuss, } \\
\text { debate }\end{array}$ & $\begin{array}{l}\text { Make recom- } \\
\text { mendations for } \\
\text { alternative } \\
\text { solutions }\end{array}$ \\
\hline
\end{tabular}

Figure 1. General steps of simulation system modeling

Simulation modeling has grown fast over the past forty years. The major paradigms that have largely influenced this field are System Dynamics (SD), Discrete Event (DE), Agent Based (AB), and Dynamic Systems (DS). The four approaches display various levels of aggregation and abstraction [15]. As opposed to SD [16] which deals with high level aggregates describing the system from a strategic point of view, DS are used in particular settings, mainly technical engineering, and provide very specific answers based on state variables and algebraic differential equations with direct "physical" meaning. This focused approach puts DS under the low abstraction level classification based on the work of Borshchev and Filippov [15]. According to the same source, DE approach [17] is based on the concept of entities, resources and block charts, and 
highlights medium abstraction levels thanks to its ability to track the general movement of objects across the system while being neither highly strategic nor too specific. Finally, AB modeling is the computational study of social agents as evolving systems of autonomous interacting agents [18]. The power of this method derives from its inherent bottom-up approach to understanding a system's behavior, allowing for a larger span of both aggregation and abstraction levels (individual agents interacting locally to give rise to a large-scale aggregated outcome).

While for each of the four paradigms a significant amount of attention has been given to the models' components and to the simulation part (phases II and III in Figure 1). However, to our knowledge, none of the existing studies has addressed in a deep enough level the link between phase I and phase II (Figure 1). In fact, the main literature skims across the system description aspect by offering three equally limited alternatives: case studies, soft operations research, and systems thinking.

Case studies depend on observation, analysis and debate. While having the advantage of bringing the problem close to reality, it displays the major drawback of being highly dependent on the personal judgment and subjective interpretation of the people studying the case. Soft operations research is fundamentally similar to the case study approach in analyzing business and social systems. While it suggests formal procedures for eliciting and organizing the system description in phase I (Figure 1), it does not address either how to translate these procedure into a dynamic system capable of simulating the problem at hand (the link between phase I and phase II). Lastly, systems thinking is a construct which appeared along side with SD and which refers summarily to the general awareness of systems and their importance in representing the real world while failing as well to accurately describe how system understanding can systematically lead to its modeling.

According to SD pioneer Forrester [19], all three alternatives remain soft methodologies with no rigorous quantitative foundation. In fact, although they utilize various organizational and presentation techniques, they still rely significantly on intuition and discussion. In addition, none of these three alternatives constitutes a solid base for rigorously constructing simulation models. Forrester [19] argues that "system dynamics [...] is lacking objective processes for model conceptualization", he adds "there is still much room for very constructive research on the process of converting information from the real world into system dynamics simulation models."

The essence of this paper resides in attempting to bridge this conceptualization gap. We will present a modeling method to help conceptualize and construct simulation models based on multicriteria decision making method DEMATEL. More particularly, We propose to apply our method in SD modeling because the latter relies most clearly on the concept of Causal Loop Diagrams (CLD), a representation of a system's cause-effect relationships, which is also the keystone result of DEMATEL technique.

\section{SySTEM Dynamics MODELING}

Because our world involves many complexities, it is not always obvious to comprehend systems and make sense of the intertwined relationships that govern them. Complexity can be combinatory, arising from the links among, and the number and scope of elements in the system, or dynamic, resulting from the dynamic aspect of those elements and thus their counterintuitive behavior over time [20]. System Dynamics Modeling brings the advantage of modeling this complexity by combining the technical grounding from mathematics and engineering with the nonlinearities of social sciences, organizational behavior, and psychology. As such, models are constructed by incorporating various elements that could affect the system either from the inside or the outside, including those variables that could wrongly be overlooked because no historical data is available, for example. Indeed, omitting such variables is equivalent to saying they have zero effect, which is probably the only value that is known to be wrong [16]. 
System dynamics modeling is based on the concept of Causal Loop Diagrams (CLD). A CLD is a graphic representation of the relationships and feedback loops among the elements of a system. In a feedback loop, a component may impact upon another one and cause it to change. The impact is transferred to other components along the loop, causing the change to occur back into the originating component [21]. In addition, when the change throughout the loop causes to strengthen the originating element, the loop is reinforcing, otherwise, it is called a balancing loop [21]. A CLD also highlights the polarity of the impact created. Positive (+) or negative (-) signs are placed next to each arrow indicating the type of impact. A positive link indicates that "if the cause increases, the effect increases above what it would otherwise have been" [20]. Likewise, a negative link indicates that "if the cause increases, the effect decreases below what it would otherwise have been" [20]. By modeling the feedback loops on a simulation software using appropriate variables and equations, it is possible to view the results of the intricate links created in the system and to simulate the future behavior of each component of the model.

Another central concept of the SD approach is the stock-flow diagram. Stocks represent the accumulations within the system, either tangible or intangible. An example of a tangible stock is inventory, or the number of trained workers. An intangible stock could be the level of employees' motivation or customer satisfaction. Flows symbolize the rate of change in the system. They are represented by inflows - which augment the level of the stock, or outflows - which reduce the level of the stock. Examples of flows are the daily production rate, or the monthly number of trained workers. When put together, stocks and flows of a system constitute the stock-flow diagram. In general, the mathematical relation between stocks and flows is represented by the following integral form [20]:

$\operatorname{Stock}(\mathrm{t})=\int_{t 0}^{t}[\operatorname{Inflow}(s)-$ Outflow $(s)] d s+\operatorname{Stock}\left(\mathrm{t}_{0}\right)$

where $t_{0}$ is the initial time, Stock $\left(\mathrm{t}_{0}\right)$ the stock level at the initial time, and $t$ the current time. $s$ indicates the change in the time variable between the initial time and the current time, Inflow $(s)$ and Outflow $(s)$ represent respectively the information coming into and going out of the stock at time $s$. In the stock-flow diagram, connectors are arrows linking the system's components to each other and transmitting information throughout the system using linear or non-linear equations (eg. Figure 2).

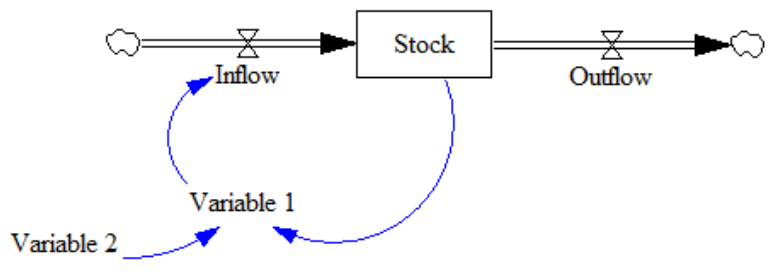

Figure 2. Example of a stock-flow chart

The SD approach brings the main advantage of helping to overcome the limitations of mental models. In fact, as advanced as our understanding of the world could be, research has shown that the human mind is unable to make sense of the intricacies that link numerous elements of a system to one another and how these links unfold in time. Controlled experimental research has confirmed the limitations of our mental models [22-24]. As Sterman [25] put it, "Our mental models are limited, internally inconsistent, and unreliable." (p.10) " Where the world is dynamic, evolving, and interconnected, we tend to make decisions using mental models that are static, narrow, and reductionist. Among the elements of dynamic complexity people find most problematic are feedback, time delays, stocks and flows (accumulations), and nonlinearity." (p.11) Both system dynamics researchers and cognitive psychologists have agreed that the human mind "cannot men- 
tally simulate any but the simplest models without error" (p.10) [26]. The response came from industry specialists and system dynamics researchers who developed computerized simulation models to help overcome the limitations of mental models-based decision making. System dynamics provided, indeed, a structured approach of conceptualizing system complexities and simulating the evolution of their intrinsic relationships over time.

However, if our mental models prevent us from simulating complex systems with an acceptable degree of accuracy, wouldn't they also be distorting our processes of constructing the business dynamics models supposed to help resolve these complexities? Could they be, for example, preventing us from realizing the very existence of some non-obvious relations among variables in the SD model? It has been shown, in fact, that it is often assumed that events have a single major cause, and as soon as this first sufficient cause is identified, people stop short of considering other potential causes [27]. Research has also shown that our representation of the world depends on the way we look at, think about, and act upon systems, typically, SD modeling depends on the modeler's points of view and understanding of the context subject to study [28] causing a subjectivity bias to occur. This bias is further emphasized when defining the model's equations, especially those related to qualitative and intangible variables for which no solid historical data might exist. For example, the extent to which team motivation might impact upon a firm's reputation in the consulting industry can differ in magnitude from a modeler to another.

Therefore, if system dynamics models are constructed based solely on decision makers' mental models and understanding of the context, then the resulting systems must necessarily bear some degree of deficiency due to the very mental models which led to these systems' conception. As such, a systematic method for constructing SD models could prove essentially helpful in overcoming the biases dictated by our limited understanding and conceptualization of complex systems.

In this paper, we propose to use the DEMATEL decision making method as an input to the construction of SD models.

\section{Proposing a NeW System Dynamics MOdeling METHOD}

In this section, we propose a combined method to support system dynamics model construction. We use the Decision Making Trial and Evaluation Laboratory (DEMATEL) technique to systematically define and quantify causal relationships among the variables of a system, then we use DEMATEL resulting Impact Relation Maps (IRMs) to construct the system dynamic's causal loop diagram. Impact measures obtained through DEMATEL's total relation matrix are used as weights in the stock-flow diagram equations. The first sub-section presents the DEMATEL method, followed in the second sub-section by the presentation of our proposed method.

\subsection{DEMATEL}

DEMATEL method was initiated by the Battelle Memorial Institute of Geneva between 1972 and 1976 as a technique to structure complex cause and effect relationships among the elements of a system. This method has been widely used over the past years and applied in various fields [13, 29-38]. In its original version, the DEMATEL method can be summa-rized as follows [36]:

First, the pair wise impact matrices of $n$ indicators are collected from a group of $p$ experts. Each expert is asked to provide his or her assessment of the influence that factors have got on one another by affecting integer scores ranging from 0 to 4 using the comparison scale in Table 1 . Let $X(k)=\left[x_{i j}(k)\right]_{n \times n}$ denote the $n \times n$ answer matrix of expert $k$. In matrix $X(k)$, the element $x_{i j}(k)$ denotes the impact that factor $i$ has got on factor $j$ according to expert $k$. 
Table 1. Comparison scale of the DEMATEL technique

\begin{tabular}{ll}
\hline Score & Meaning \\
\hline 0 & No influence \\
1 & Low influence \\
2 & Medium influence \\
3 & High influence \\
4 & Very high influence \\
\hline
\end{tabular}

The resulting experts' answer matrices $X(1), X(2), \ldots X(p)$ are averaged out in order to obtain the $n \times n$ average matrix $A=\left[a_{i j}\right]_{n \times n} . A$ is calculated by averaging out the experts' scores as in Equation (2):

$a_{i j}=\frac{\sum_{k=1}^{p} x i j(k)}{p}$

Second, the normalized initial direct matrix $D$ is obtained by multiplying the elements of average matrix $A$ by the number $\lambda$ as explained in Equation (3) and (4). The resulting matrix is the normalized initial direct matrix $D$.

$D=\lambda * A$
$\lambda=\operatorname{Min}\left[\frac{1}{\operatorname{Max} 1 \leq i \leq n \sum_{j=1}^{n}|a i j|}, \frac{1}{\operatorname{Max} 1 \leq j \leq n \sum_{i=1}^{n}|a i j|}\right]$

Third, the total direct/indirect influence matrix $T=\left[t_{i j}\right]_{n x n}$ is obtained by raising matrix $\mathrm{D}$ to an infinite power, which guarantees the continuous decrease of indirect effects along the powers of $\mathrm{D}$ and the convergence of the total direct/indirect matrix $T$ to the inverse matrix $T=D(I-D)^{-1}$ as (Equation 5):

$$
\begin{aligned}
\lim _{m \rightarrow \infty} D^{m}=[0]_{n \times n} \\
T=D+D^{2}+D^{3}+\ldots+D^{m} \\
=D\left(I+D+D^{2}+\ldots+D^{m-1}\right)(I-D)(I-D)^{-1} \\
=D(I-D)^{-1} \quad \text { where } I \text { denotes the identity matrix. }
\end{aligned}
$$

However, this method has been contested in the study conducted by [14] who argued that the normalized initial direct-indirect matrix $D$ does not necessarily converge to the null matrix, meaning that $\lim _{m \rightarrow \infty} D^{m}=[0]_{n \times n}$ might or might not be true. In fact, the study has proven that the convergence is only possible under the sufficient condition that the column sum of each column of the initial direct-indirect matrix $D$ is less than one. To correct for this limitation, a modification is made to normalizing factor $\lambda$ by introducing the number $\varepsilon$, a very small positive number such that (Equation6):

$\lambda^{\prime}=\operatorname{Min}\left[\frac{1}{\operatorname{Max} 1 \leq i \leq n \sum_{j=1}^{n}|a i j|}, \frac{1}{\varepsilon+\operatorname{Max} 1 \leq j \leq n \sum_{i=1}^{n}|a i j|}\right]$

With this modification, it is possible to correct for the original DEMATEL while making sure that the total influence matrix $T$ converges to the inverse matrix in Equation4 (Tzeng et al., 2013). In addition, vectors $\mathrm{R}$ and $\mathrm{C}$ are defined such that: 


$$
\begin{aligned}
& R=\left[r_{i}\right]_{n \times 1}=\left[\sum_{j=1}^{n} t_{i j}\right]_{n \times 1} \\
& C=\left[c_{j}\right]_{l \times n}=\left[\sum_{i=1}^{n} t_{i j}\right]_{l \times n}
\end{aligned}
$$

The sum $r_{i}$ of the $i^{\text {th }}$ row of matrix $T$ denotes the total direct and indirect influence that factor $i$ exerts on all other factors of the system, while the sum of the $j^{\text {th }}$ column of matrix $T, c_{i}$, shows the direct and indirect influence that factor $j$ has received from all other factors of the system. In addition, when $i=j, r_{i}+c_{i}$ indicates the central role that factor $i$ plays in the system while $r_{i}-c_{i}$ reveals whether factor $i$ is a net dispatcher of impact, when $r_{i}-c_{i}$ is positive, or a net receiver of impact, when $r_{i}-c_{i}$ is negative.

Finally, a graphical representation of the causal relationships is made using an Impact Relation Map (IRM) which translates values of the total influence matrix $T$ into directed graphs or digraphs (eg. Figure 3). Factors are represented in a graph using their $\left(r_{i}+c_{i}, r_{i}-c_{i}\right)$ values to show the various relations among them. For example, in Figure 3, factor $f_{1}$ influences both $f_{2}$ and $f_{3}$ with an impact strength of 3 and 2 respectively. In order to reduce the complexity of the resulting IRM, a threshold value $\alpha$ is set for matrix $T$, helping to keep only values greater than $\alpha$, those deemed most important for the analysis.

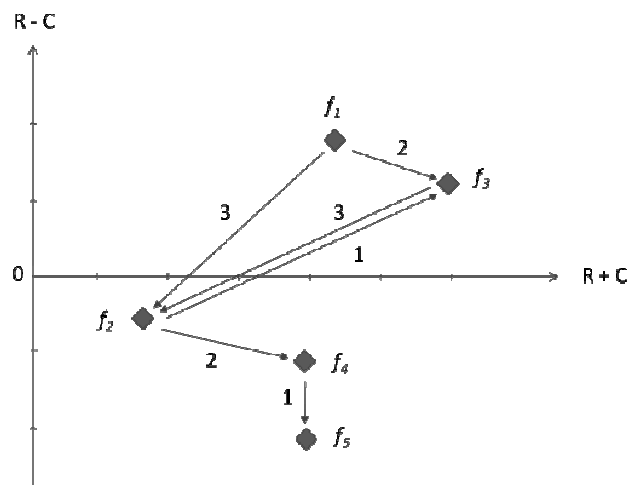

Figure 3. Example of an Impact Relation Map

\subsection{The proposed SD modeling Method}

In the standard SD approach, Causal Loop Diagrams (CLD) are built based on experience and understanding of the context and the domain subject to study. In this paper, it is proposed that the construction of the feedback loops is further enriched by the outcomes of DEMATEL technique. Cause and effect diagrams, or IRMs, are translated into partial CLDs where relations between factors are weighted using the DEMATEL total effect values calculated from the total-influence matrix $T$. The stepwise process for the proposed method can be structured as described in Figure 4.

- Step 1: Determine the domains of study. The objective of this first step is to define the project's boundaries and determine the research areas that are most relevant to the study;

- Step 2: Define indicators by domain. For every domain, a list of performance indicators is set by a team of experts;

- Step 3: Construct partial IRMs. Use revised DEMATEL to derive a relation diagram per domain of study. The resulting IRMs will help distinguish between the cause factors, or dispatchers, and the effect factors, or receivers, for every domain. This process will also give an indication about which performance indicators deserve more careful attention; 
- Step 4: Derive partial Causal Loop Diagrams (CLD). Build a CLD per domain based on the resulting DEMATEL digraphs. Those have a two-fold benefit: 1) they help systematically determine the causal relationships which should be taken into account in the SD model construction. These relations are then readily translated into CLDs; and 2) they help obtain quantitative impact values which are used in the stock-flow diagram equations to weigh the degree of influence of a factor over another;

- Step 5: Construct the integrated CLD. Build the integrated CLD by combining all CLDs of topical domains using overlapping variables/indicators. It is important to mention that for overlapping variables, the calculation of the percent impact values should be based on the total impact received from all variables of the system; which entails the use of data from the total influence matrices of all domains of study.

- Step 6: Build the Stock Flow Chart and Define Equations. The integrated stock-flow chart is built based on the obtained CLD from Step 5. Equations are defined using the values from the DEMATEL total influence matrices. More precisely, for each domain, the total influence matrix is translated into a weighting matrix using column values and column totals. The impact that each factor receives from other factors is translated into percent impact values.

- Step 7: Analyze and Conclude. Check the model, test it, and run simulations on the integrated CLD. At this stage, the modeler can build various scenarios and analyze the long term evolution of the system under each scenario, which could provide a powerful tool for helping to recommend the best strategic choice. 
International Journal of Computer Science \& Information Technology (IJCSIT) Vol 7, No 2, April 2015

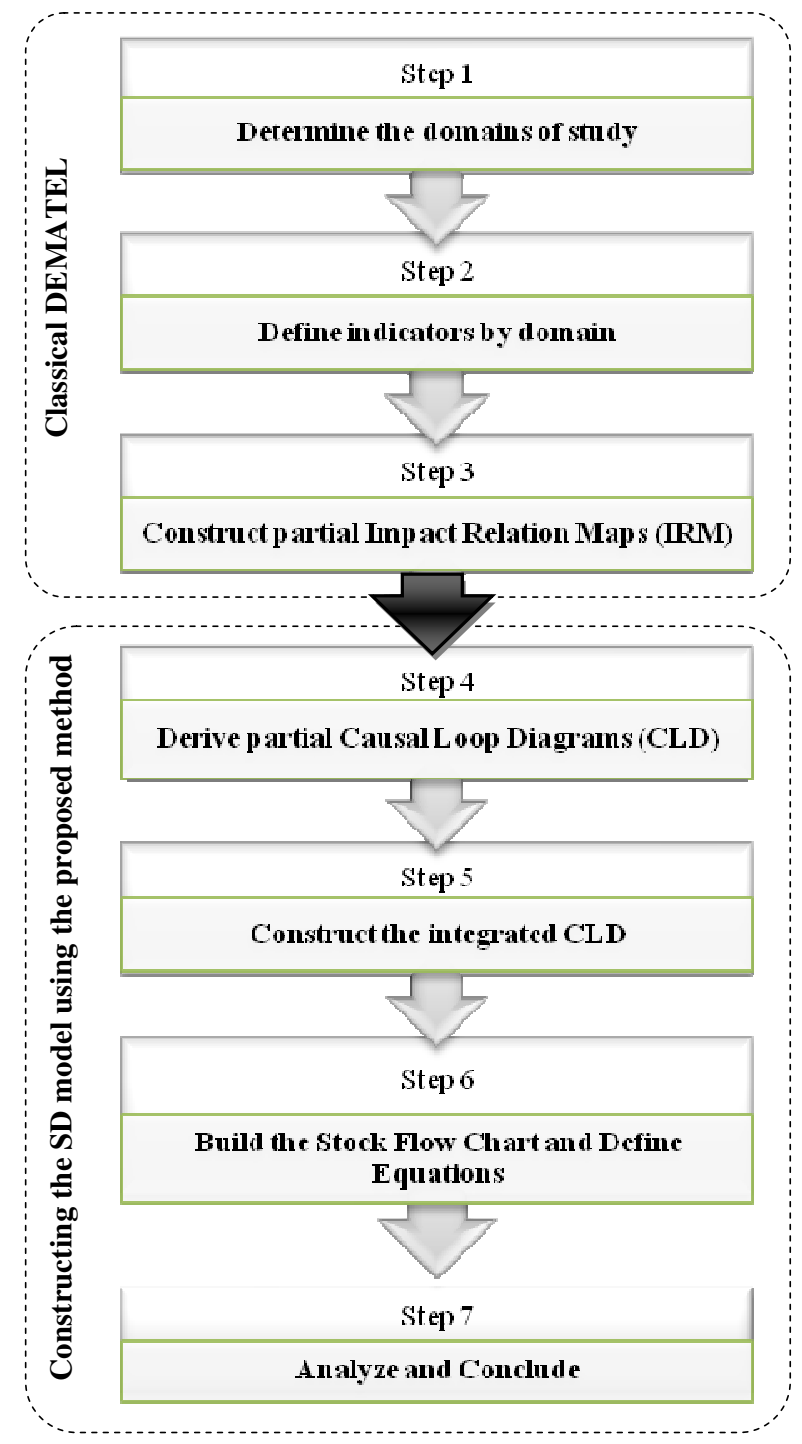

Figure 4. Stepwise process of the proposed method

\subsection{Illustration and Discussion}

To bring further clarification to the above method, let us use a simple illustration. In the human resources management domain, let Motivation $\left(A_{1}\right)$, Training Effectiveness $\left(A_{2}\right)$, and Recognition $\left(A_{3}\right)$ be the factors subject to study. Assuming that DEMATEL results show that factor $A_{1}$ is impacted by $A_{2}$ and $A_{3}$ with the respective values 0.2 and 0.3 in the total-influence matrix $T$, then in percent terms, the impact that factor $A_{l}$ is receiving from the two factors respectively is $40 \%$, and $60 \%$ (eg. Figure 5). The resulting CLD should highlight links from $A_{2}$ and $A_{3}$ toward $A_{1}$, and the calculated percent impact values will serve as weights for variables in the stock-flow chart in order to more accurately quantify the degree of impact (eg. Figure 5).

If, in addition, the modeler recognizes that these factors feature a linear relationship with each other -either by experience or available data- then the equation for $A_{l}$ could be written as (Equation 8): 
In the absence of the proposed DEMATEL weighting approach, the modeler would have simply written $A_{l}$ as (Equation 9): $A_{l}=A_{2}+A_{3}$

which confers factors equal influence power and which, therefore, fails to consider the instance where a factor is a major cause of impact as compared with another. As such, in the illustrated example, Motivation would be defined as (Equation 10) :

Motivation $=0.4 \times$ Training Effectiveness $+0.6 \times$ Recognition

Let us assume that, in addition, Motivation $\left(A_{1}\right)$ is an overlapping variable receiving additional impact from the economic factors Turnover $\left(B_{1}\right)$ and Workload $\left(B_{2}\right)$ with the respective values of 0.4 and 0.3 . If these are the only impact relations detected by DEMATEL and whose values are higher than threshold $\alpha$, then the new impact weights for Motivation $\left(A_{1}\right)$ will be $17 \%, 25 \%, 33 \%$ and $25 \%$ respectively produced by $A_{2}, A_{3}, B_{1}$, and $B_{2}$. Therefore, the equation for factor $A_{1}$ would be (Equation 11):

$A_{1}=0.17 \times A_{2}+0.25 \times A_{3}+0.33 \times B_{1}+0.25 \times B_{2}$

It could be assumed in this example that Turnover $\left(B_{1}\right)$ is positively correlated with Motivation $\left(A_{1}\right)$. As for Workload $\left(B_{2}\right)$, it could be assumed that, if it is less or equal to 1 (i.e. $\left.100 \%\right)$, then it can lead to positive motivation, which would be translated by a positive correlation between $A_{I}$ and $B_{2}$. Otherwise, excess workload (with value greater than $100 \%$ ) may decrease motivation, leading to a negative correlation. The equation for factor $A_{l}$ could then be written as follows (Equation 12):

If $\left(B_{2}<=1\right)$

Then $A_{1}=0.17 \times A_{2}+0.25 \times A_{3}+0.33 \times B_{1}+0.25 \times B_{2}$

Else $A_{1}=0.17 \times A_{2}+0.25 \times A_{3}+0.33 \times B_{1}-0.25 \times B_{2}$

This proposed weighting approach can similarly be used in non-linear equations and shows particular importance when such intangible indicators are involved in the model as client satisfaction, reputation, team cohesion...etc.

For the present illustration, the steps described in Section 4.2 (eg. Figure 4) are summarized in Figure 5. Each IRM is directly translated into the corresponding CLD, then into an integrated CLD employing overlapping variable $A_{l}$. Then, total influence matrices' values for all five variables are calculated on a percent basis to give rise to percent total values which will be used in the stock-flow chart's equations. The model is built on VENSIM ${ }^{\circledR}$ software and simulation results are presented (eg. Figure 5). Results show that Training Effectiveness $\left(A_{2}\right)$ will improve following a hyperbolic shape -thanks to the training effectiveness goal-gap loop- which will in turn be transmitted to Motivation $\left(A_{l}\right)$. This pattern is not alleviated by the impact of Turnover $\left(B_{l}\right)$ which is an increasing linear function resulting of the linear inflow and outflow of Workload $\left(B_{2}\right)$. Finally, it is worth mentioning that the illustration above is a plain simplification of a real-life setting. In an improved version of it, Training Effectiveness $\left(A_{2}\right)$ would be represented by the outcomes of a survey or an empirical study, and Turnover $\left(B_{1}\right)$ would not depend solely on Workload $\left(B_{2}\right)$ but on as varied revenue driving factors as the number of shifts, the production capacity...etc. Obviously, other variables impact directly upon Motivation $\left(A_{l}\right)$ such as the salary, the work environment, future career prospects...etc.

\footnotetext{
${ }^{1}$ In this example, it is assumed that Motivation is positively correlated with both Training Effectiveness and Recognition.
} 
Therefore, the simplification adopted in this paper conveys mostly an objective of highlighting our proposed method than a deficiency in modeling the various variables.

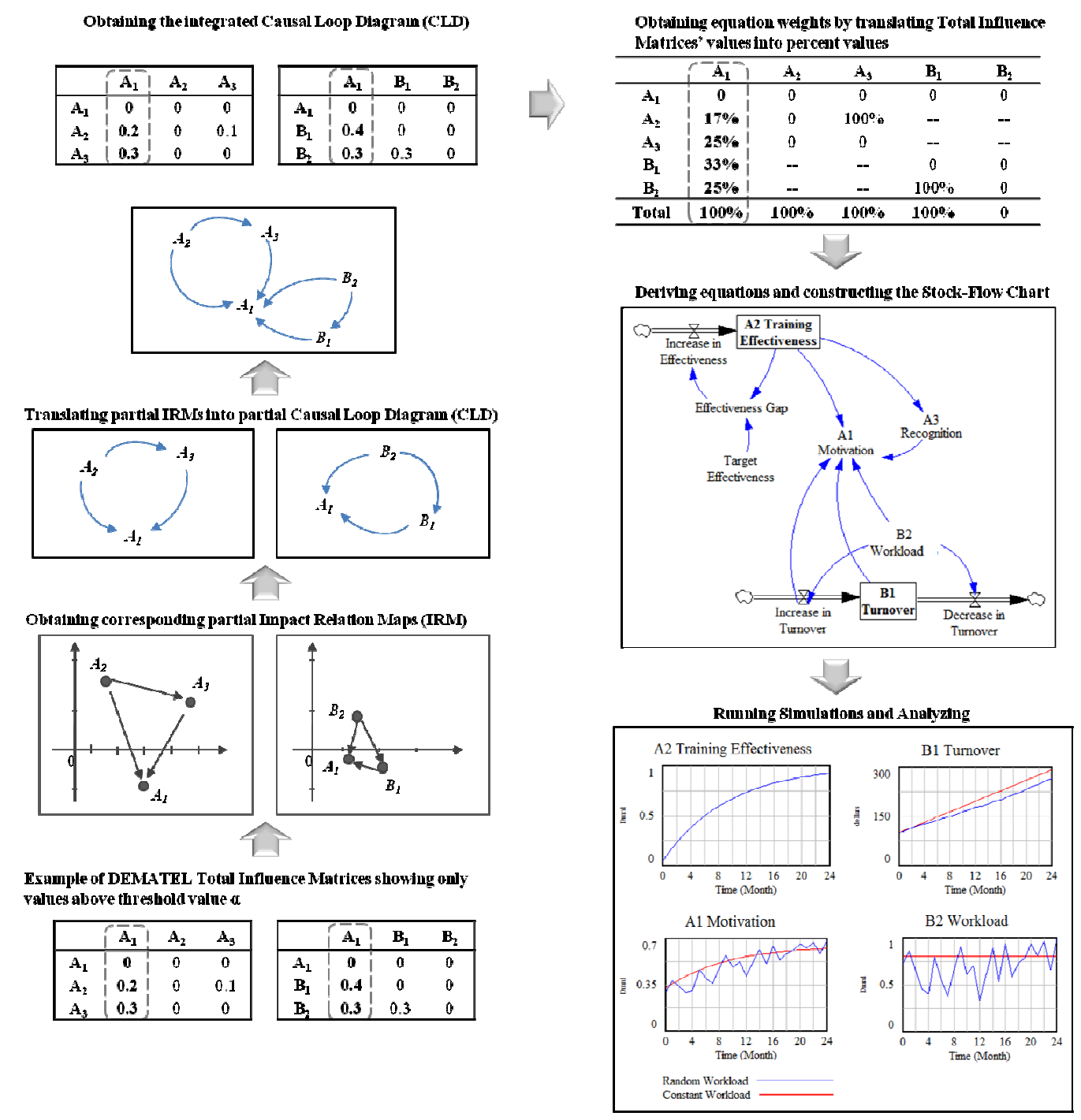

Figure 5. An illustration of the proposed Method

We believe that our proposed method adds value to both DEMATEL technique and the System Dynamics approach. On the one hand, using the standard SD modeling alone to construct models might present the limitation of overlooking some important but non-trivial relationships that could exist between variables and that the modeler may unwillingly ignore. On the other hand, as interesting as DEMATEL technique is, it provides fixed results whose evolution in time could be hardly predictable without substantial margin of error, especially with complex systems involving numerous indicators. More specifically, by combining these two approaches, four main advantages are met:

1. Firstly, the use of a scientific method to support model construction helps to reduce the subjectivity bias [28] caused by the human mental models' limitations associated with the stan- 
dard SD approach. In this sense, our method directly contributes to bridging the conceptualization gap identified in Section 2.

2. Secondly, the identification of new links and loops between variables, thanks to DEMATEL IRMs, forces managers to consider options they might not have previously thought of, which helps expand their imagination and bolster their grasp of the business.

3. Thirdly, the use of DEMATEL outcomes as variable weights in the stock-flow chart equations brings the model closer to reality by enhancing the model's representation of the realworld, which proves critical especially when dealing with qualitative variables with little or no historical data.

4. Finally, this combined approach allows to take DEMATEL approach into a dynamic simulation mode which offers an appropriate environment for managers to reflect on scenarios, conduct analyses, and make the right strategic choices.

\section{CONCLUSION AND PERSPECTIVES}

As useful as System Dynamics (SD) modeling has proved to be in helping decision makers analyze complex systems' progress and in guiding their strategic policy making choices, the SD approach continues to bear some limitations due mainly to the human mind's narrow mental models and the resulting subjectivity bias. This paper proposes a systematic method for constructing SD models using Decision Making Trial and Evaluation Laboratory (DEMATEL) technique. DEMATEL is a Multi-Criteria Decision Making (MCDM) framework used to identify cause-effect relationships within the variables of a system. In this research, it is proposed that DEMATEL's Impact Relation Maps (IRMs) be translated into Causal Loop Diagrams (CLDs) in a systematic fashion. Furthermore, it is suggested that the outcome values of DEMATEL's Total influence matrix be used as input weights in the stock-flow chart's equations of the SD model.

While the proposed approach combines the advantages of both SD and DEMATEL and overcomes some of their critical shortcomings, it still recalls the modeler's careful sense of judgment, particularly in defining the model's equations. In fact, if this approach systematically indicates the existence of impact links between variables and the scope of that impact -indicating those factors which deserve closer attention as compared with others-, it does not inform of the nature of the relationships between and among variables, namely linearity or non-linearity, in which sense the modeler's best judgment, experience and expertise are required. This particular feature adds, in turn, more value to the proposed method as it does not exclude the modeler's input and offers, instead, more room for customized modeling.

This research would be further enriched by an application to a real experimental case study we are working on in order to emphasize the significance of the proposed method in a more practical way.

\section{REFERENCES}

[1] Govindan, K., et al., Multi criteria decision making for green supplier evaluation and selection: a literature review. Journal of Cleaner Production, 2013. x: p. 1-18.

[2] Wu, C. and D. Barnes, A literature review of decision-making models and approaches for partner selection in agile supply chains. Journal of Purchasing \& Supply Management, 2011. 17: p. 256-274.

[3] Chai, J., J.N.K. Liu, and E.W.T. Ngai, Application of decision-making techniques in supplier selection: A systematic review of literature. Expert Systems with Applications, 2013. 40: p. 3872-3885.

[4] Ho, W., X. Xu, and P.K. Dey, Multi-criteria decision making approaches for supplier evaluation and selection: A literature review. European Journal of Operational Research, 2010. 202: p. 16-24.

[5] Lin, C.-T., C.-B. Chen, and Y.-C. Ting, An ERP model for supplier selection in electronics industry. Expert Systems with Applications, 2011. 38: p. 1760-1765.

[6] Wu, D., Supplier selection: A hybrid model using DEA, decision tree and neural network. Expert Systems with Applications, 2009. 36: p. 9105-9112. 
[7] Shaw, K., et al., Supplier selection using fuzzy AHP and fuzzy multi-objective linear programming for developing low carbon supply chain. Expert Systems with Applications, 2012. 39: p. 8182-8192.

[8] Dong, X., et al., Application of a system dynamics approach for assessment of the impact of regulations on cleaner production in the electroplating industry in China. Journal of Cleaner Production, 2012. 20(1): p. 72-81.

[9] Machado, R.R., et al., Evaluation of forest growth and carbon stock in forestry projects by system dynamics. Journal of Cleaner Production, 2013: p. 1-11.

[10] Georgiadis, P. and M. Besiou, Sustainability in electrical and electronic equipment closed-loop supply chains: A System Dynamics approach. Journal of Cleaner Production, 2008. 16(15): p. 1665-1678.

[11] Saysel, A.K., Y. Barlas, and O. Yenigün, Environmental sustainability in an agricultural development project: a system dynamics approach. Journal of Environmental Management, 2002. 64(3): p. 247260.

[12] Lee, S., et al., Dynamic and multidimensional measurement of product-service system (PSS) sustainability: a triple bottom line (TBL)-based system dynamics approach. Journal of Cleaner Production, 2012. 32: p. 173-182.

[13] Lin, C.-L. and G.-H. Tzeng, A value-created system of science (technology) park by using DEMATEL. Expert Systems with Applications, 2009. 36(6): p. 9683-9697.

[14] Lee, H.-S., et al., Revised DEMATEL: Resolving the Infeasibility of DEMATEL. Applied Mathematical Modelling, 2013. 37(10-11): p. 6746-6757.

[15] Borshchev, A. and A. Filippov. From system dynamics and discrete event to practical agent based modeling: reasons, techniques, tools. in Proceedings of the 22nd international conference of the system dynamics society. 2004.

[16] Forrester, J.W., Industrial Dynamics1961, Cambridge, MA, USA: MIT Press.

[17] Gordon, G. A general purpose systems simulation program. in Proceedings of the December 12-14, 1961, eastern joint computer conference: computers-key to total systems control. 1961. ACM.

[18] Janssen, M.A. and E. Ostrom, Empirically based, agent-based models. Ecology and Society, 2006. 11(2): p. 37.

[19] Forrester, J.W., System dynamics, systems thinking, and soft OR. System Dynamics Review, 1994. 10(2-3): p. 245-256.

[20] Sterman, J., Business Dynamics: Systems Thinking and Modeling for a Complex World2000, Boston, MA, USA: Irwin/McGraw-Hill.

[21] Hannon, B. and M. Ruth, Dynamic Modelling1994, New York, USA: Springer-Verlag.

[22] Brehmer, B., Dynamic decision making: Human control of complex systems. Acta Psychologica, 1992. 81(3): p. 211-241.

[23] Kleinmuntz, D.N., Information processing and misperceptions of the implications of feedback in dynamic decision making. System Dynamics Review, 1993. 9(3): p. 223-237.

[24] Sterman, J.D., Modeling managerial behavior: Misconceptions of feedback in dynamic decision making. Organizational Behavior and Human Decision Processes, 1989. 43(3): p. 301-335.

[25] Sterman, J.D., System Dynamics Modeling: Tools for Learning in a Complex World. California Management Review, 2001. 43(4).

[26] Doyle, J.K. and D.N. Ford, Mental models concepts for system dynamics research. System Dynamics Review, 1998. 14(1): p. 3-29.

[27] Plous, S., The Psychology of Judgment and Decision Making1993, New York, NY, USA: McGraw Hill.

[28] Vázquez, M. and M. Liz, Models as Points of View: The Case of System Dynamics. Foundations of Science, 2011. 16(4): p. 383-391.

[29] Diabat, A., R. Khodaverdi, and L. Olfat, An exploration of green supply chain practices and performances in an automotive industry. The International Journal of Advanced Manufacturing Technology, 2013. 68(1-4): p. 949-961.

[30] Horng, J.-S., et al., Creativity as a critical criterion for future restaurant space design: Developing a novel model with DEMATEL application. International Journal of Hospitality Management, 2013. 33: p. 96-105.

[31] Govindan, K., D. Kannan, and K.M. Shankar, Evaluating the drivers of corporate social responsibility in the mining industry with multi-criteria approach: A multi-stakeholder perspective. Journal of Cleaner Production, 2014: p. 1-19.

[32] Shaik, M.N. and W. Abdul-Kader, Comprehensive performance measurement and causal-effect decision making model for reverse logistics enterprise Computers \& Industrial Engineering, 2014. 68: p. 87-103. 
[33] Chen, F.-H., T.-S. Hsu, and G.-H. Tzeng, A balanced scorecard approach to establish a performance evaluation and relationship model for hot spring hotels based on a hybrid MCDM model combining DEMATEL and ANP. International Journal of Hospitality Management, 2011. 30(4): p. 908-932.

[34] Hsu, C.-W., et al., Using DEMATEL to develop a carbon management model of supplier selection in green supply chain management. Journal of Cleaner Production, 2013. 56: p. 164-172.

[35] Tsai, W.-H. and J.-L. Hsu, Corporate social responsibility programs choice and costs assessment in the airline industry-A hybrid model. Journal of Air Transport Management, 2008. 14(4): p. 188-196.

[36] Yang, Y.-P.O., et al., A Novel Hybrid MCDM Model Combined with DEMATEL and ANP with Applications International Journal of Operations Research, 2008. 5(3): p. 160-168.

[37] Tsai, W.-H. and W.-C. Chou, Selecting management systems for sustainable development in SMEs: A novel hybrid model based on DEMATEL, ANP, and ZOGP. Expert Systems with Applications, 2009. 36(2): p. 1444-1458.

[38] Tsai, W.-H., et al., An integrated approach for selecting corporate social responsibility programs and costs evaluation in the international tourist hotel. International Journal of Hospitality Management, 2010. 29(3): p. 385-396.

\section{Authors}

\section{Mohammed Abdou Janati Idrissi}

Pr. Mohammed Abdou Janati Idrissi holds a Ph.D. in Mathematics and Computer Science. He is Adjunct Directror of Academic Affairs, Head of TIME (Information Technology and Enterprise Management) research team, and Head of e-MBI (eManagement and Business Intelligence) academic program at ENSIAS (National Higher School for Computer Science and System Analysis) Engineering School, Rabat Mohammed V University, Morocco. Prior to that, Prof. Janati was Head of Computers and Decision Aid Department at ENSIAS. His research focuses on decision-aid methods, networks optimization and project management. In addition to his academic work, Prof. Janati provides expertise services to a range of project man-

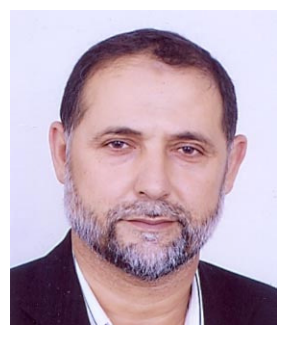
agement, decision aid, and business intelligence projects for many large companies. His works are published in a number of management information systems and computer science academic journals, conference publications, and books.

\section{Abdellah El Manouar}

Pr. Abdellah El Manouar holds a Ph.D. in Economics from Montreal University, Canada. He is Head of the Business Intelligence concentration of e-MBI program, and member of TIME research team at ENSIAS (National Higher School for Computer Science and System Analysis) Engineering School, Rabat Mohammed V University, Morocco. Before, Prof. El Manouar was Head of Computers and Decision Aid Department at ENSIAS. Prof. El Manouar's research interests cover finance and investment, management, net-economy, financial management and financial engineering. He delivers courses in financial valuation, portfolio management and e-management.

\section{Fadwa Chaker}

Fadwa Chaker is currently a Ph.D. candidate at ENSIAS (National Higher School for Computer Science and System Analysis), Rabat Mohammed V University, Morocco. Her research thesis focuses on decision aid modeling and simulation systems applied to Corporate Social Responsibility. Ms. Chaker obtained a National Engineering Diploma and a Master of Computer Science Engineering from ENSIAS Engineering School in 2004, and an MBA in Decision Sciences and Strategy from Duke University on a CMS Energy-Fulbright Scholarship (2007 - 2009). Ms.

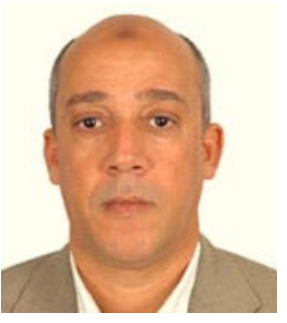
Chaker has worked as a consultant in business intelligence, corporate performance management, and organizational strategy. 